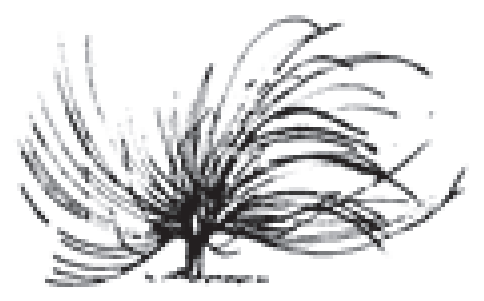

\title{
Constructivismo en los Procesos de Educación en Línea
}

\author{
Carlos Vargas López ${ }^{1}$ \\ Instituto Nacional de Aprendizaje (INA) \\ Heredia, Costa Rica \\ cvargas@cpcecr.com \\ Susana Isabel Jiménez Sánchez ${ }^{2}$ \\ Universidad Nacional \\ Heredia, Costa Rica \\ susana.jimenez.sanchez@una.cr
}

\begin{abstract}
Resumen
El constructivismo como teoría pedagógica en la que se enmarcan los procesos de enseñanza y de aprendizaje ha fundamentado la práctica educativa presencial, pero ¿Será aplicable en la enseñanza y el aprendizaje en línea? Esta pregunta llevó a la elaboración del presente artículo, por lo que se realizó una revisión de la literatura con respecto del constructivismo: definición, ventajas, desventajas, aplicabilidad en la educación en línea. Si bien no hay un concepto único de constructivismo y existen académicos que consideran que no tiene uso real para la
\end{abstract}

Recibido: 12 de setiembre de 2013 -Aprobado: 25 de octubre de 2014

1 Doctorando en: Instructional Technology and Distance Education, by Nova Southeastern University, USA. Magíster en Administración de Negocios, por la Universidad de Costa Rica. Labora como docente en: Escuela Ciencias de la Administración, UNED, y en el Instituto Nacional de Aprendizaje (INA), Regional Heredia.

2 Doctora en Educación con énfasis en Instructional Technology and Distance Education, de Nova Southeastern University, USA. Máster en Tecnología Educativa de la Universidad Estatal a Distancia. Licenciada en Enseñanza de la Química de la Universidad de Costa Rica y en Ciencias de la Educación con énfasis en Docencia de la Universidad Estatal a Distancia. Con estudios en Educación Primaria. Actualmente es académica de la División de Educología del Centro de Investigación y Docencia en Educación (CIDE) de la UNA. 
enseñanza y el aprendizaje; es esa pluralidad la que le da amplias posibilidades de empleo en la educación línea.

Palabras clave: constructivismo, educación en línea, práctica pedagógica, didáctica, psicología educativa

\begin{abstract}
Constructivism, as a pedagogical theory in which teaching and learning processes are framed, has been the basis for face-to-face educational practice, but is it applicable to teaching and learning online? This question was the starting point for this article. Thus, a literature review about constructivism-its definition, advantages, disadvantages, and applicability in online education was conducted. Even though there is a single definition for constructivism, there are scholars who believe it has no real use for teaching and learning; it is this plurality that gives it wide possibilities of usage in online education.
\end{abstract}

Keywords: constructivism, online education, pedagogical practice, didactics, educational psychology

\title{
Introducción
}

$\mathrm{E}$ n una práctica educativa tradicionalista, el estudiante se presenta como actor pasivo. La pasividad más que formar, deforma, como lo ha señalado el poeta Facundo Cabral: "Yo fui inteligente hasta que entré a la escuela" (Rodríguez, 2003, p. 83). Ser constructivista es ser educador desde la cátedra, saber trabajar interdisciplinariamente. Educar significa trabajar en función de construir un mundo mejor, donde los seres humanos nos responsabilicemos por formar personas, desde la sabiduría del pensar en el otro, hablar y razonar con inteligencia, manteniendo como objetivo alcanzar las metas propuestas (Fumero, 2001).

G.Hernández (2008) manifiesta que el conocimiento no es una copia fiel de la realidad, pero en cambio, sí es una auténtica construcción relativista sobre la realidad existente. Al respecto, Piaget proponía experiencias intelectuales esquematizadas; Kant mencionaba que estos esquemas son estáticos y preformados, sin embargo, la corriente constructivista va más allá de la filosofía kantiana, al poner a prueba estas ideas en los procesos de enseñanza y de aprendizaje, 
determinando así que nuestro aprendizaje evoluciona y se fortalece con nuestras vivencias.

El constructivismo como teoría pedagógica ha fundamentado la práctica educativa presencial, pero, ¿será aplicable en la enseñanza y el aprendizaje en línea? A partir de esta pregunta se elaboró el presente trabajo, para lo que se realizó una revisión de literatura con respecto del constructivismo. Este escrito se ha estructurado en cuatro apartados sobre el constructivismo: definición, ventajas, desventajas y aplicabilidad en la educación en línea.

\section{¿Qué es el constructivismo?}

Para el constructivismo, el conocimiento se construye y se almacena a través de esquemas o modelos mentales, que van cambiando, agrupándose y volviéndose más sofisticados, utilizando para ello dos procesos complementarios: la asimilación y el alojamiento (Piaget, 1955, citado por G.Hernández, 2008). Asimismo, la actividad intelectual se nutre desde los distintos procesos cognitivos (percepción, aprendizaje, comprensión de textos, etc.), permitiendo generar predicciones y la elaboración de inferencias. Pese a tener variables que permiten una poderosa capacidad de adaptación en el sujeto, son muy estables en el tiempo, de modo que algunos han criticado ampliamente su estaticidad, proponiendo un nuevo constructo: los modelos mentales.

El conocimiento organiza las experiencias intelectuales de forma esquemática, sin embargo, al contrario de la filosofía kantiana, estos esquemas no son estáticos ni pre-formados, sino que se trata de evoluciones cíclicas a partir de la actividad que realicen con objetos físicos por medio de la acción (la parte motriz), la relación social (el aspecto afectivo) y lo intelectual, se relaciona con el nivel de conciencia con lo que se hace (Londoño, 2008).

La teoría clásica menciona al constructivismo como una doctrina, la cual impulsa al estudiante a desarrollar un rol activo en la búsqueda de su conocimiento y la comprensión de éste, dotando de sentido a la información adquirida. Este conocimiento es construido individualmente, en interacción simultánea con el medio social, y teniendo como plataforma las nociones sobre el tema adquiridas previamente (Woolfolk, 1999). 
El constructivismo se ha transformado en una nominación genérica que involucra desde la propuesta original de Piaget y Kant, hasta la incorporación de teorías como las de Vygotsky y Wallon. Esto ha llevado a algunos constructivistas a distinguir sus posiciones y diferentes enfoques por medio de términos tales como: constructivismo social, constructivismo exógeno, constructivismo endógeno, constructivismo radical, constructivismo epistémico y constructivismo dialéctico, como parte del andamiaje construido por teóricos e investigadores, los cuales han aportado sus diferentes teorías o propuestas (Miranda, 2000). En la tabla 1 se sintetizan algunos postulados teóricos de algunos tipos de constructivismo.

El constructivismo lleva a concebir y desarrollar los procesos de enseñanza y de aprendizaje desde los conocimientos previos del individuo, sus experiencias de vida y la posible aplicabilidad de los contenidos aprendidos en el contexto real de las personas. Esto es válido en todos los contextos educativos, pero es aún más importante para quienes son mediadores de procesos educativos con adultos, pues tal afirmación implica, además, conocer la realidad del estudiante para adaptar la enseñanza a sus necesidades, esto es, aplicando los principios didácticos de la andragogía.

Ahora bien, tampoco debemos escudarnos en la andragogía y el constructivismo para actuar de forma imprudente: el estudiante con formación andragógica debe realizar las actividades educativas en orden, con normas, considerando el respeto al profesor o a la institución educativa. La absoluta flexibilidad no existe; el estudiante se evalúa objetivamente y no se sobreevalúa.

Las teorías andragógica y constructivista tampoco pueden ser el discurso legitimador de la comercialización y pragmatización de la educación. Es decir, no se deben desarrollar cursos y talleres sin soporte académico, sin seguimiento de los procesos de aprendizaje y sin un propósito educativo. Este propósito no sólo debe considerar a la utilidad individual de los procesos, sino que debe pensarse y proponerse desde la pertinencia con la realidad social y con el compromiso de transformación (Rodríguez, 2003).

Rojas (2008) menciona con certeza, que los estudiantes tienen la suficiente capacidad de aprender estrategias cognitivas, motivacionales, afectivas y de autorregulación, al interactuar con la reflexión 


\section{Tabla 1. Síntesis de los postulados teóricos de diferentes tipos de} constructivismo

\begin{tabular}{l|l}
\hline $\begin{array}{l}\text { Tipo de } \\
\text { constructivismo }\end{array}$ & Postulados teóricos \\
\hline $\begin{array}{l}\text { Constructivismo } \\
\text { social }\end{array}$ & $\begin{array}{l}\text { Enfatiza en el esfuerzo de dar soluciones coherentes a los proble- } \\
\text { mas de enseñanza y aprendizaje, puesto que en él se desarrolla la } \\
\text { idea de una perspectiva social de la cognición, así como el análi- } \\
\text { sis del conocimiento en estrecha interacción con los contextos que } \\
\text { se usan, por lo que no es posible separar los aspectos cognitivos, } \\
\text { emocionales y sociales presentes en el contexto en el que se actúa. }\end{array}$ \\
\hline $\begin{array}{l}\text { Constructivismo } \\
\text { exógeno }\end{array}$ & $\begin{array}{l}\text { El sujeto reconstruye su realidad elaborando redes conceptuales a } \\
\text { través de representaciones precisas. Se construyen estructuras men- } \\
\text { tales exactas que reflejan la realidad desde el contexto del sujeto. }\end{array}$ \\
\hline $\begin{array}{l}\text { Constructivismo } \\
\text { endógeno }\end{array}$ & $\begin{array}{l}\text { Se moldea el mundo externo a través del conocimiento que se } \\
\text { construye en las estructuras cognoscitivas, las cuales se vuelven } \\
\text { útiles para el desarrollo cognoscitivo del sujeto. Su principal repre- } \\
\text { sentante es Piaget. }\end{array}$ \\
\hline $\begin{array}{l}\text { Constructivismo } \\
\text { radical }\end{array}$ & $\begin{array}{l}\text { Establece que el conocimiento no se recibe pasivamente, ni a tra- } \\
\text { vés de los sentidos, ni por medio de la comunicación, sino que es } \\
\text { construido activamente por el sujeto cognoscente, de manera que } \\
\text { la función de la cognición es adaptativa y sirve a la organización } \\
\text { del mundo experiencial del sujeto, no al descubrimiento de una } \\
\text { realidad ontológica objetiva. }\end{array}$ \\
\hline $\begin{array}{l}\text { Constructivismo } \\
\text { epistémico }\end{array}$ & $\begin{array}{l}\text { El ser humano no es un receptáculo pasivo o un ente meramente } \\
\text { reactivo, sino que lo que se conoce es el producto de la actividad } \\
\text { cognitiva, experiencial o subjetiva del sujeto. El conocimiento es } \\
\text { altamente dependiente del sujeto, de su actividad y del contexto en } \\
\text { donde éste se genera. }\end{array}$ \\
$\begin{array}{l}\text { Admite que el conocimiento se construye como resultado de las re- } \\
\text { laciones entre factores de carácter: interno y externo, ambientales y } \\
\text { sociales. Las bases de su desarrollo se deben a Vigotsky. }\end{array}$ \\
\hline
\end{tabular}

Nota: Elaboración con base en Barreto, Gutiérrez, Pinilla y Parra (2006), G. Hernández (2008) y S. Hernández (2008).

metacognitiva. Por eso, una didáctica constructivista debe proponer estrategias de enseñanza que lleven al estudiante a cuestionarse el cómo aprende. 


\section{¿Qué ventajas tiene el aplicar el constructivismo en los procesos educativos?}

Efectivamente, las propuestas sobre el construccionismo realizadas por importantes investigadores indican que estas teorías tienen ventajas importantes para el desarrollo intelectual de los estudiantes. Barreto, Gutiérrez, Pinilla y Parra (2006), mencionan un aspecto importante: la formación y orientación de los estudiantes a desarrollar la cualidad de crear una actitud proactiva hacia el aprendizaje; esto lleva a una cognición adaptativa que le sirve al estudiante como punto de apoyo a su mundo de experiencias cognitivas. Un principio que se realza del constructivismo es el planteamiento de que el estudiante desarrolla su aprendizaje, en un viaje que lo lleva de lo más complejo a lo más sencillo. Este proceso lo llevará a descifrar una amplia gama de conocimientos complejos y abstractos sobre la vida como un todo.

La aproximación estratégica busca promover en los estudiantes la toma de conciencia de lo que han aprendido y de los procesos que requieren para autorregularse y alcanzar dichos aprendizajes. Cuando se les enseña a los estudiantes a utilizar las estrategias cognitivas autorreguladoras y la reflexión metacognitiva, lo que se trata es de que lleguen a alcanzar de forma intencional sus objetivos, es decir, que éstos aprendan a construir su forma personal de aprender (S. Hernández, 2008).

El constructivismo ha provisto una serie importante de constructos teóricos, de gran significancia para el proceso educacional en general y de importante familiaridad para los involucrados en dicho proceso; algunos de estos constructos que se pueden mencionar son los siguientes: aprender a aprender, aprendizaje significativo, aprendizaje colaborativo, adaptaciones curriculares, autoaprendizaje, comunidades de aprendizaje, ritmos de aprendizaje, pertinencia curricular, metodologías cualitativas (Cabrera, 2005).

La coordinación de experiencias motoras (es otro punto dentro de las ventajas que se debe considerar), hace que los niños acepten o rechacen determinados objetos al desarrollar mejor su destreza manual, de forma que se logra desarrollar la cognición superior, esto es confirmado con la visión constructivista, la cual hace énfasis en la construcción del conocimiento, al mejorar sus nociones didácticas y metodológicas que desembarcan en la noción del aprendizaje significativo (Barreto et al. 2006). 


\section{¿Cuáles son las desventajas del constructivismo?}

El constructivismo goza de un número importante de seguidores en el campo de la educación y el aprendizaje, pero también tiene sus detractores; esto indica que posee aspectos importantes a rescatar, así como elementos que se deben observar con detenimiento, con el propósito de evitar encontrarse en una calle sin salida. A continuación se exponen algunos aspectos que se pueden considerar como desventajas del constructivismo, y que se deben de tener en consideración.

Según se indica en Barreto et al. (2006), el constructivismo, desde la perspectiva epistemológica y pedagógica de sus detractores, no es una teoría de enseñanza. Esto plantea problemas epistemológicos, debido a que es difícil determinar un objeto de estudio propio del constructivismo, lo que imposibilita explicar cuál es el papel del docente y cómo ejercen su influencia en los estudiantes ya que es el estudiante quien construye por sí mismo su conocimiento.

Continuando con las desventajas del constructivismo, se menciona también su estimulación hacia el individualismo ya que propugna el relativismo personal, como percepción de su visión y entendimiento de su principal propuesta, que es dirigida al objeto de estudio, el cual es utilizado como plataforma para la construcción de su conocimiento. Considerando que lo anterior genera cierta construcción subjetiva al reducir el realismo en función a aspectos mecanicistas y contemplativos, por lo tanto, es imperativo evitar tomar a la ligera los postulados del constructivismo, con el propósito de evitar caer en generalizaciones (Barreto et al. 2006).

\section{¿Cuál es la aplicabilidad del constructivismo en la educación en línea?}

Las tecnologías de la información y la comunicación (TIC) las conforman todos aquellos dispositivos o medios digitales que permiten extender nuestras capacidades físicas y mentales, así como nuestro nivel de socialización. De acuerdo con Marques (2000), el concepto de las TIC se interpreta como "los medios de comunicación social (mass media)" y los medios de comunicación interpersonales tradicionales. Para S.Hernández (2008), las TIC son responsables en gran medida del desarrollo de la globalización económica, así como de la rapidez con la 
que se suscitan los cambios. Asimismo, continúa S.Hernández, se tiene por demostrado que los profesores constructivistas fomentan en sus estudiantes la utilización de las TIC de cara a sus actividades escolares.

El impacto de las TIC en la sociedad está generando una atomización de opciones para participar de los diferentes procesos de enseñanza y de aprendizaje en línea, por lo que la educación se está transformando con el uso de estas diferentes opciones de herramientas, algunas que nos brinda el mercado con fines comerciales y económicos, entre ellas: a) medios masivos de comunicación: televisión, radio y Internet; b) recursos: computadora, red, proyector multimedia, pizarrón electrónico, videoconferencias; y c) aplicaciones como la web, chat, correo electrónico y foros en línea, wikis, redes sociales, blogs, entre otras (Marques, 2000).

Del constructivismo se puede sustraer un componente clave: la enseñanza se dirige a realizar tareas auténticas; estas tienen relevancia y utilidad dentro del contexto de cada realidad. Los estudiantes tienen la oportunidad de ampliar su experiencia cognoscitiva al utilizar las nuevas tecnologías como herramientas para un aprendizaje constructivista (S.Hernández, 2008).

Los medios, recursos y aplicaciones han revolucionado las aulas y la presencialidad dándole un giro impresionante y han propiciado el surgimiento de técnicas educativas en apoyo a la enseñanza, como la videoconferencia, los cursos en línea y los materiales educativos tales como los objetos de aprendizaje (OA). En los sistemas de educación superior que cuenten con Universidad Virtual ya es posible obtener un bachillerato, licenciaturas, maestrías y doctorados en línea (Marques, 2000).

Esto trae sus propias ventajas para quienes logran incorporarse a este sistema y tener éxito e implica compromisos para los estudiantes y administrativos que trabajan en línea. Se mencionan problemas como: la deserción, la falta de motivación y un sentimiento de soledad en los estudiantes o los problemas de orden institucional, como uno de los hechos para dar el mismo reconocimiento que merece a los estudios en línea (Marques, 2000).

S.Hernández (2008) señala que los roles más importantes en el proceso enseñanza y de aprendizaje han sufrido innovaciones en tres aspectos: 1) su naturaleza; 2) el lugar y la forma donde se realiza; 3) el papel a desempeñar por los estudiantes y los docentes en tal proceso. La Web 2.0 (que permite la colaboración en línea) forma parte de las 
nuevas tecnologías que han trastocado estos importantes roles, específicamente, en el punto dos de los mencionados antes.

Tim O’Reilly, quien desarrolló este concepto, lo define así: la red como plataforma, que abarca los hardware de conexión; las aplicaciones de la Web 2.0 son aquellas que hacen el mayor uso de las ventajas intrínsecas de esa plataforma: entregar software como un servicio continuamente actualizado, que mejora cuantas más personas lo utilicen, consumiendo y reutilizando datos de múltiples fuentes, incluyendo usuarios individuales, mientras proporcionan sus propios datos y servicios de una manera que permite que otros la vuelvan a combinar, estableciendo un efecto de red a través de una "arquitectura de participación", y partiendo más allá de la página metáfora de la Web 1.0 para suministrar a los usuarios una experiencia fructífera (S.Hernández, 2008).

\section{Reflexiones finales}

Los aportes de investigadores sobre el constructivismo como disciplina psicoeducativa, así como su implementación teórico/práctica en los educandos, ha enriquecido a una gran cantidad de estudiantes, con un conjunto de nuevos conceptos y formatos teóricos que han redireccionado de forma importante al análisis, la reflexión y el desarrollo de propuestas innovadoras de los procesos de aprendizaje, enseñanza, diseño, evaluación y desarrollo curricular (Hernández, 2008).

Es posible afirmar, de acuerdo con lo expuesto por Castillo (2008), que las TIC han cambiado el paradigma de los diseños informáticos. En la época actual, el resultado de la integración de los multimedios con las comunicaciones, con la computadora como herramienta fundamental y la Web conceptualizada como vía de acceso a un espacio de interacción social diferente a lo anteriormente conocido, permite concebir a un interlocutor, como un sujeto de acción en un mundo en el que ocurren comunicaciones tanto con objetos reales como virtuales. Esto es redefinir el rol de la tecnología, pues se transforma en un soporte al proceso de enseñanza que sirve para mejorar el aprendizaje.

Como reflexión final, apoyada en el criterio emitido por Rigo (2008), es importante concluir que el profesor debe reflexionar sobre cómo alcanzar su objetivo: una didáctica con orientación constructivista. Sin embargo, también debe reflexionar de manera constructiva. La propuesta se orienta hacia el terreno de la realimentación de su propio 
trabajo: el docente debe reinventarse como aprendiz, autodidacta, o bien, como un especialista proactivo, esto es, como participante fundamentado, cooperativo, crítico, comprensivo y creativo, con el propósito de poner en práctica los principios constructivistas, que luego ha de ejemplificar a sus estudiantes.

Quedó corroborado en lo expuesto anteriormente, que lo fundamental de los principios formativos del constructivismo (que durante muchos años ha estado presencialmente vigente, en las aulas de escuelas, colegios, universidades y de muchos recintos donde se lleva a cabo procesos de aprendizaje y de enseñanza), está listo para continuar su desarrollo por medio del ciberespacio, a través de procesos de educación en línea contextualizados y no masificados.

Para lograrlo, es de vital importancia establecer dentro del currículo de las diferentes asignaturas que se van a ofrecer en línea, la normativa de que los docentes deben incorporar en sus actividades para los estudiantes tareas, proyectos, investigaciones, entre otras, basadas en información obtenida dentro de su familia, comunidad, centro educativo o empresas, con el claro objetivo de que realicen la construcción de su conocimiento, a partir de actividades que les pongan en contacto con la realidad de su entorno.

\section{Referencias bibliográficas}

Barreto, C., Gutiérrez, L. F., Pinilla, B y Parra, C. (2006). Límites del constructivismo pedagógico. Revista Educación y Educadores, 9 (1), 11-31.

Cabrera, F. (2005). Evaluación, constructivismo y metacognición. Aproximaciones teórico-practicas. Revista Horizontes Educacionales, 10, 27-35.

Castillo, S. (2008). Propuesta pedagógica basada en el constructivismo para el uso óptimo de las tic en la enseñanza y el aprendizaje de la matemática. Revista latinoamericana de investigación en matemática educativa, 11 (2), 171-194.

Fumero, F. (2001). El arte de educar desde el constructivismo. Revista Educere, 13, (5), 86.

Hernández, G. (2008). Los constructivismos y sus implicaciones para la educación. Revista Perfiles educativos, 30 (122), 38-77. 
Hernández, S. (2008). El modelo constructivista con las nuevas tecnologías: Aplicado en el proceso de aprendizaje. En Comunicación y construcción del conocimiento en el nuevo espacio tecnológico. Revista de Universidad y Sociedad del Conocimiento, 5 (2), 26-35.

Londoño, C. (2008): Avatares del Constructivismo: de Kant A Piaget. Revista Historia de la Educación Latinoamericana, 10 (1), 73-96.

Marquès, P. (2000). Las TIC y sus aportaciones a la sociedad. Recuperado de http://dewey.uab.es/pmarques/tic.htm

Miranda, M. (2000). El constructivismo como principio explicativo en la educación: Una pretensión y un riesgo. EDUCERE, 10 (4), 8-16.

Rigo, M. A. (2008). Constructivismo educativo, actividad y evaluación del docente: Relato de algunas posibles incongruencias. Revista Reencuentro, 53, 125-134.

Rodríguez, P. (2003). La andragogía y el constructivismo en la sociedad del conocimiento. Revista de Educación Laurus, 9, (15), 80-89.

Woolfolk, A. (1999). Psicología educativa. (7a ed.). México: Prentice Hall. 